\title{
Design and Synthesis of Some 5-Substituted-2-(4-(azido or methylsulfonyl)phenyl)-1H-indole Derivatives as Selective Cyclooxygenase (COX-2) Inhibitors
}

\author{
Afshin ZARGHI ${ }^{*}{ }^{1}$, Azar TAHghighI ${ }^{1}$, Zohreh Soleimani ${ }^{1}$, \\ Bahram Daraie ${ }^{2}$, Orkideh Gorban Dadrass ${ }^{3}$, Mehdi Hedayati ${ }^{4}$ \\ ${ }^{1}$ Department of Medicinal Chemistry, School of Pharmacy, \\ Shahid Beheshti University (M.C), P.O. Box: 14155-6153, Tehran/ Iran \\ ${ }^{2}$ Department of Toxicology, School of Medical Sciences, \\ Tarbiat Modares University, Tehran/ Iran \\ ${ }^{3}$ School of Pharmacy, Azad University, Tehran/ Iran \\ ${ }^{4}$ Endocrine research center, Shahid Beheshti University (M.C), Tehran/ Iran
}

\begin{abstract}
A group of 5-substituted-2-(4-azido or (methylsulfonyl)phenyl)- $1 H$-indoles were designed and synthesized as selective cyclooxygenase (COX-2) inhibitors. In vitro COX-1 and COX-2 isozyme inhibition studies were carried out to investigate the effect of different substituents $(\mathrm{H}, \mathrm{F}, \mathrm{Cl}, \mathrm{Me}, \mathrm{OMe})$ at $\mathrm{C}-5$ position and different pharmacophore groups (azido or methylsulfonyl) at para position of phenyl ring at C-2 position of the $1 \mathrm{H}$-indole ring on COX-2 selectivity and potency. The structureactivity relationship study of these compounds indicated that the introduction of a methoxy substituent at C-5 position and 4-(methylsulfonyl) phenyl group at C-2 position of the $1 \mathrm{H}$-indole ring (compound $4 \mathrm{e}$ ) had the best COX-2 selectivity (S.I = 291.2). A molecular modeling study where $4 \mathrm{e}$ was docked in the binding site of COX-2 showed that the methylsulfonyl group at para position of phenyl ring is oriented in the vicinity of the COX-2 secondary pocket.
\end{abstract}

\footnotetext{
* Corresponding author: Fax: +98-21-88795008.

E-mail: azarghi@yahoo.com (A. Zarghi).
} 


\section{Keywords}

2-Phenyl-1H-indoles $•$ COX-2 inhibitors $• \mathrm{SAR}$

\section{Introduction}

Cyclooxygenase (COX) also know as prostaglandin synthase (PGH) is a potent mediator of inflammation. Nonsteroidal anti-inflammatory drugs (NSAIDs) bind to cyclooxygenase thereby inhibiting the production of prostaglandins. However, inhibition of COXs may lead to undesirable side effects. Nowadays, it is well established that there are at least two COX isozymes, COX-1 and COX-2 [1]. The constitutive COX-1 isozyme is produced in a variety of tissues and appears to be important to the maintenance of physiological functions such as gastric protection and vascular homeostasis [2]. Alternatively, the COX-2 isozyme is induced by mitogenic and proinflammatory stimuli linking its involvement to inflammatory processes [3].Thus, selective inhibition of COX-2 over COX-1 is useful for the treatment of inflammation and inflammation-associated disorders with reduced gastrointestinal toxicities when compared with NSAIDs. In addition to the role of COX-2 in rheumatoid arthritis and osteoarthritis, it is also implicated in colon cancer and angiogenesis [4,5]. Recent studies have shown that the progression of Alzheimer's disease is reduced among some users of NSAIDs. Chronic treatment with selective COX-2 inhibitors may therefore slow the progress of Alzheimer's disease without causing gastrointestinal damage [6]. Diarylheterocycles, and other central ring pharmacophore templates, have been extensively studied as selective COX-2 inhibitors. All these molecules possess 1,2 diaryl substitution on a central heterocyclic or carbocyclic ring system (see structures A-F in Chart 1) [7-13]. Recently, we reported several investigations describing the design, synthesis and COX inhibitory activities of a novel class of compounds possessing an acyclic 1,3diarylprop-2-en-1-one structural template [14, 15]. For example, the acyclic $(E)$ 1,3diphenylprop-2-en-1-ones possessing a 4-methylsulfonyl or 4-azido COX-2 
pharmacophore group at the C-1 phenyl ring (see structure F) exhibited highly selective COX-2 inhibition.

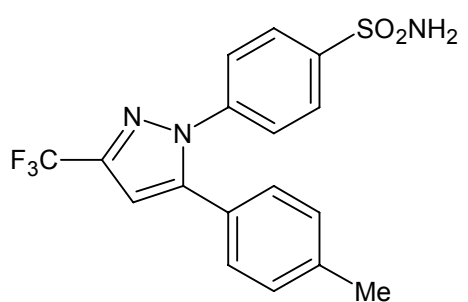

Celecoxib (A)

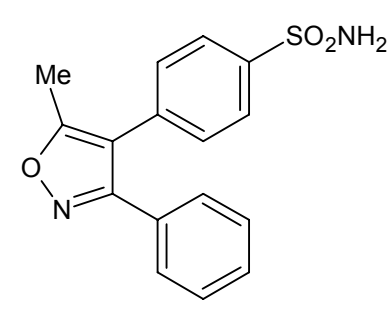

Valdecoxib (C)

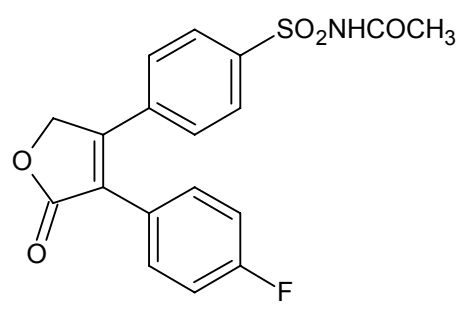

E

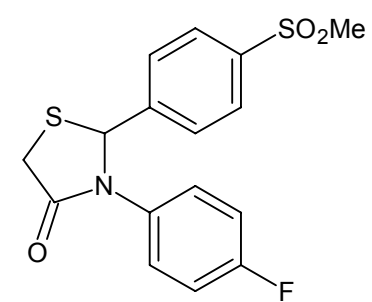

(B)

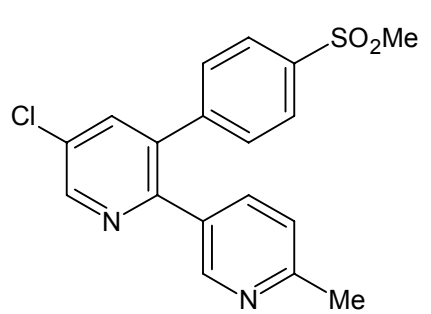

Etoricoxib (D)

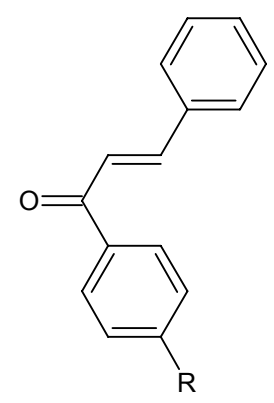

$\mathbf{F}\left(\mathrm{R}=\mathrm{SO}_{2} \mathrm{Me}\right.$ or $\left.\mathrm{N}_{3}\right)$

Chart 1. Representative examples of selective COX-2 inhibitors

As part of our ongoing program to design new types of selective COX-2 inhibitors, we now report the synthesis, some structure-activity relationships, and a molecular modeling study for a group of 2-phenyl-1H-indoles possessing a COX-2 azido or $\mathrm{SO}_{2} \mathrm{Me}$ pharmacophore at the para-position of phenyl ring in conjunction with an $1 \mathrm{H}$-indole ring having different substituents at $\mathrm{C}-5$ position. 


\section{Results and Discussion}

\section{Chemistry}

The target 2-(4-(methylsulfonyl or azido)phenyl)-1H-indole derivatives were synthesized using a Fischer-indole synthesis as shown in Scheme 1. Accordingly, warming an ethanolic mixture of 4-methylsulfonylacetophenone (1) [16] and an appropriate phenylhydrazine (2) on a water bath in presence of few amounts of acetic acid gave 4-methylsulfonylacetophenone phenylhydrazone (3) (50-89\%). Cyclization of 3 by polyphosphoric acid at $120^{\circ} \mathrm{C}$ afforded the 2-(4-(methylsulfonyl) phenyl)-1H-indoles $(4,60-92 \%)$ [17, 18]. Similarly, 2-(4-aminophenyl)-1H-indole derivatives 5 (40-62\%) were synthesized and the amino group was converted to azido group by diazotation with sodium nitrite and hydrochloric acid and then treatment with sodium azide to obtain 2-(4-(azido)phenyl)-1H-indoles 6 (55-68\%) [15]. The purity of all products was determined by thin layer chromatography using several solvent systems of different polarity. All compounds were pure and stable. The compounds were characterized by ${ }^{1} \mathrm{H}$ nuclear magnetic resonance, infrared, mass spectrometry and $\mathrm{CHN}$ analysis.

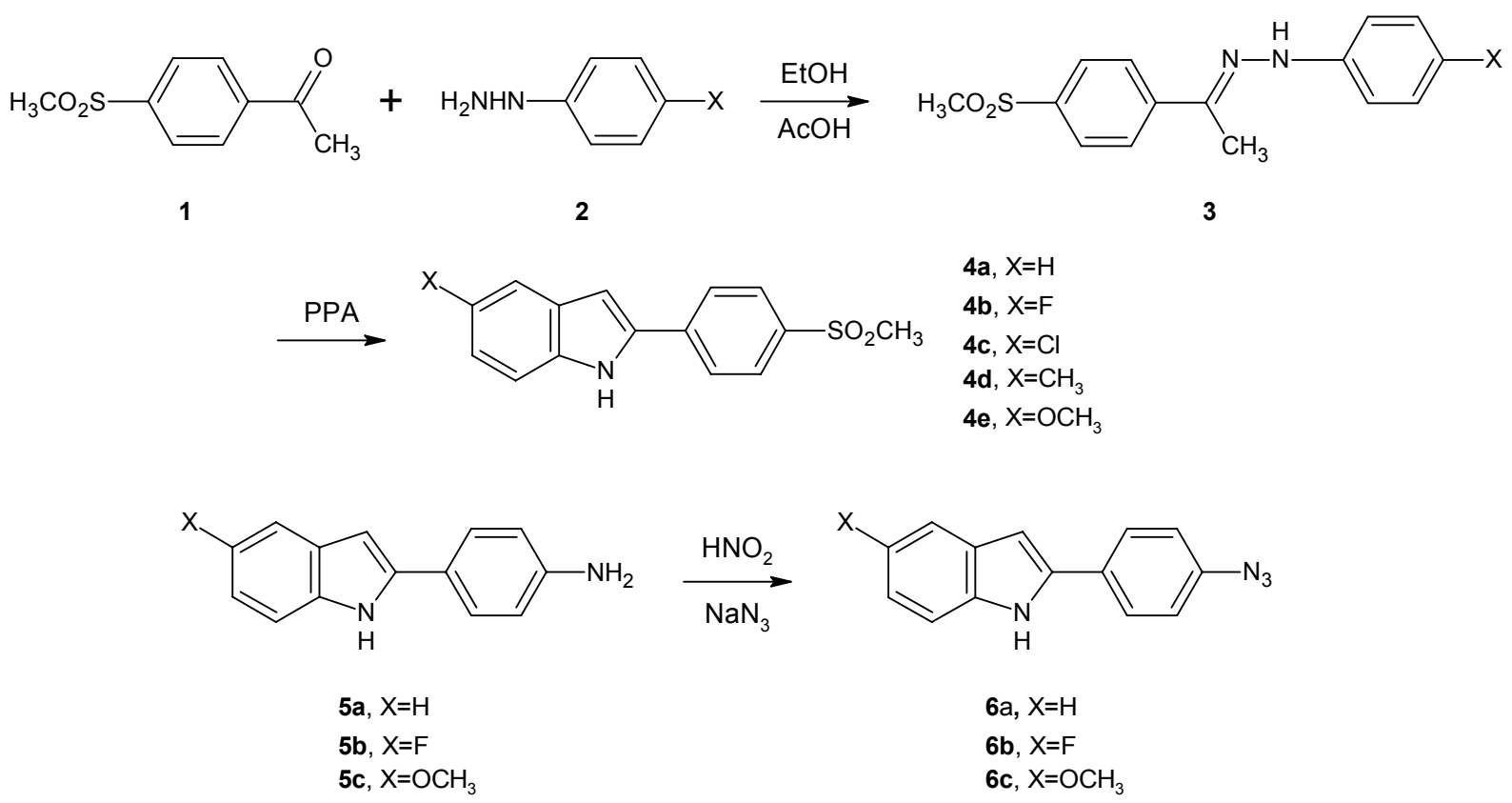

Sch. 1. 


\section{Enzyme Inhibitory Activity}

The ability of the 2-phenyl-1H-indoles $\mathbf{4 a - e}$ and $\mathbf{6 a}-\mathbf{c}$ to inhibit the COX-1 and COX-2 isozymes was determined using chemiluminescent enzyme assays as previously described [13] (see enzyme inhibition data in Table 1.). In vitro COX$1 /$ COX-2 inhibition studies showed that compounds containing methylsufonyl pharmacophore group 4a-e were selective inhibitors of the COX-2 isozyme with $\mathrm{IC}_{50}$ values in the highly potent 0.08 to $0.26 \mu \mathrm{M}$ range, and COX-2 selectivity indexes (S.I.) in the 30.5 to 291.2 range. The structure-activity relationship study of these compounds indicated that the order of $\mathrm{COX}-2$ selectivity was $\mathrm{OMe}>\mathrm{F}>\mathrm{Me}, \mathrm{Cl}$ $>\mathrm{H}$. These results showed that the nature of substituent at $\mathrm{C}-5$ position of the $1 \mathrm{H}-$ indole ring has an important role on selectivity and potency. Replacing the methylsulfonyl group with an azido pharmacophore group in these compounds (6a$6 c)$ decreased either selectivity or potency. According to these results, 5-methoxy2-(4-(methylsulfonyl) phenyl)-1H-indole $4 \mathrm{e}$ was the most potent $\left(\mathrm{IC}_{50}=0.08 \mu \mathrm{M}\right)$, and selective (S.I. >291) COX-2 inhibitor among the synthesized compounds. These data suggest that the compound $4 \mathrm{e}$ should inhibit the synthesis of inflammatory prostaglandins via the cyclooxgenase pathway at sites of inflammation and be devoid of ulcerogenicity due to the low COX-1 inhibitory activity. 
Tab. 1. In vitro COX-1 and COX-2 Enzyme Inhibition Data

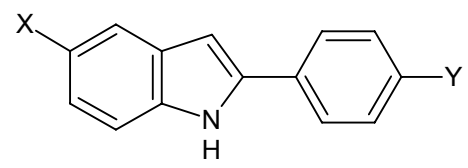

\begin{tabular}{|c|c|c|c|c|c|}
\hline \multirow[t]{2}{*}{ Compound } & \multirow[t]{2}{*}{$\mathbf{X}$} & \multirow[t]{2}{*}{$\mathbf{Y}$} & \multicolumn{2}{|c|}{$I_{50}(\mu M)^{a}$} & \multirow{2}{*}{$\begin{array}{c}\text { COX-2 } \\
\text { S.I. }^{b}\end{array}$} \\
\hline & & & COX-1 & COX-2 & \\
\hline $4 a$ & $\mathrm{H}$ & $\mathrm{SO}_{2} \mathrm{CH}_{3}$ & 7.9 & 0.26 & 30.5 \\
\hline $4 b$ & $\mathrm{~F}$ & $\mathrm{SO}_{2} \mathrm{CH}_{3}$ & 12.5 & 0.10 & 125 \\
\hline $4 c$ & $\mathrm{Cl}$ & $\mathrm{SO}_{2} \mathrm{CH}_{3}$ & 20.1 & 0.22 & 91.3 \\
\hline 4d & $\mathrm{Me}$ & $\mathrm{SO}_{2} \mathrm{CH}_{3}$ & 13.5 & 0.14 & 96.4 \\
\hline $4 e$ & OMe & $\mathrm{SO}_{2} \mathrm{CH}_{3}$ & 23.3 & 0.08 & 291.2 \\
\hline $6 a$ & $\mathrm{H}$ & $\mathrm{N}_{3}$ & 10.8 & 4.25 & 2.54 \\
\hline $6 b$ & $F$ & $\mathrm{~N}_{3}$ & 10.5 & 1.85 & 5.68 \\
\hline $6 c$ & OMe & $\mathrm{N}_{3}$ & 15.5 & 1.62 & 9.57 \\
\hline Celecoxib & & & 24.3 & 0.06 & 405 \\
\hline \multicolumn{6}{|c|}{$\begin{array}{l}{ }^{a} \text { Values are mean values of two determinations acquired using an ovine } \\
\text { COX-1/COX-2 assay kit, where the deviation from the mean is }<10 \% \text { of } \\
\text { the mean value. } \\
{ }^{\mathrm{b}} \text { In vitro COX-2 selectivity index (COX-1 IC } \mathrm{C}_{50} / \mathrm{COX}-2 \mathrm{IC} \mathrm{C}_{50} \text { ). }\end{array}$} \\
\hline
\end{tabular}

\section{Docking study}

The orientation of the highly potent and selective COX-2 inhibitor, 5-methoxy2-(4-(methylsulfonyl)phenyl)-1H-indole $4 \mathrm{e}$ in the COX-2 active site was examined by a docking experiment (Fig. 1) [18]. This molecular modeling shows that it binds in the primary binding site such that the $\mathrm{C}-2$ para- $\mathrm{SO}_{2} \mathrm{Me}$ substituent inserts into the secondary pocket present in COX-2. One of the O-atoms of $p-\mathrm{SO}_{2} \mathrm{Me}$ forms a hydrogen binding interaction with hydroxyl group $(\mathrm{OH})$ of $\mathrm{Tyr}^{355}$ (distance $=4.8 \AA \hat{\text { }}$ ). The $\mathrm{N} H$ of the central $1 H$-indole ring forms a hydrogen bond (distance $=3.4 \AA$ ) with the $\mathrm{C}=\mathrm{O}$ group of $\mathrm{Val}^{349}$. It addition, the oxygen atom of methoxy substituent at C-5 position of the $1 H$-indole ring can form hydrogen binding interaction with hydroxyl group $(\mathrm{OH})$ of $\mathrm{Tyr}^{348}$ (distance $<6 \AA$ ) which may explain the high potency of compound $4 \mathrm{e}$ compared with other derivatives. These observations together with 
experimental results provide a good explanation for the potent and selective inhibitory activity of $\mathbf{4 e}$.

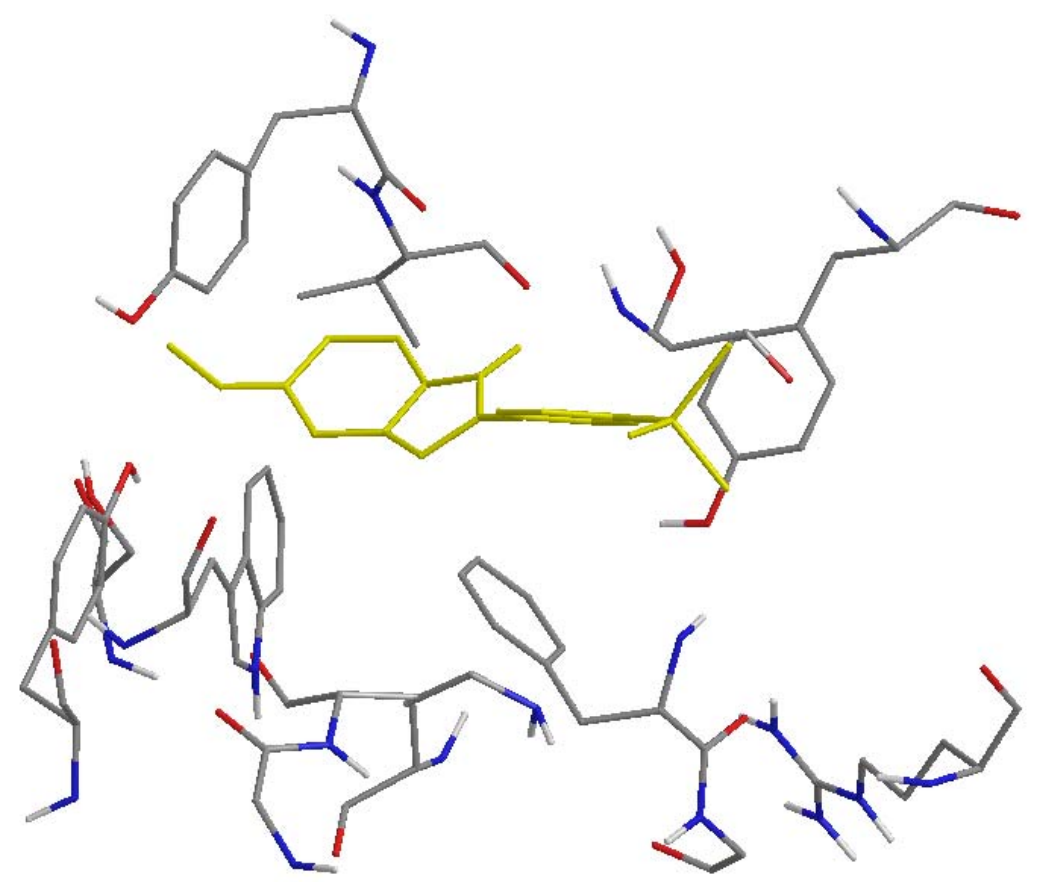

Fig. 1. Compound 4e 5-methoxy-2-(4-(methylsulfonyl)phenyl)-1H-indole docked in the active site of murine COX-2 isozyme.

\section{Experimental}

\section{Chemistry}

Melting points $(\mathrm{mp})$ were determined using a Thomas Hoover capillary apparatus (Philadelphia, USA). Infrared spectra were acquired on a Perkin-Elmer 1420 ratio recording spectrometer. A Bruker FT-500 MHz instrument (Brucker Biosciences, USA) was used to acquire ${ }^{1} \mathrm{HNMR}$ spectra; chloroform-D, DMSO- $\mathrm{D}_{6}$ and methanol- $\mathrm{D}_{4}$ were used as solvents. Mass spectra were acquired with a Finnigan TSQ-70 mass spectrometer. Electron-impact ionization was performed at an ionizing energy of $70 \mathrm{eV}$; the source temperature was $250{ }^{\circ} \mathrm{C}$. Elemental analyses were carried out with a Perkin Elmer Model 240-C apparatus (Perkin Elmer, Norwalk, CT, USA). The results of the elemental analyses $(\mathrm{C}, \mathrm{H}, \mathrm{N})$ were 
within $\pm 0.4 \%$ of the calculated amounts. All chemicals and reagents were obtained from Aldrich (USA) or Merck (Germany) and were used without further purification.

General procedure for preparation of (E)-1-(4-substituted-phenyl)-2-(1-(4(methylsulfonyl)phenyl)ethylidene)hydrazines (3)

An ethanolic mixture of 4-methylsulfonylacetophenone $1(4 \mathrm{mmol})$ and an appropriate phenylhydrazine $2(4 \mathrm{mmol})$ was heated on a water bath in presence of few amounts of acetic acid for 1 hour to give 4-methylsulfonyl acetophenone phenylhydrazone 3 . The obtained product was separated, dried and recrystallized from ethanol (Yields: $50-89 \%$ ).

(1E)-1-\{1-[4-(Methylsulfonyl)phenyl]ethylidene\}-2-phenylhydrazine (3a)

Yield: 89\%; mp: $175^{\circ} \mathrm{C}$; IR (KBr): v $\left(\mathrm{cm}^{-1}\right) 3310(\mathrm{NH}), 1300,1150\left(\mathrm{SO}_{2}\right)$, Mass, $\mathrm{m} / \mathrm{z}$ (\%): 288.3 (M+, 70), 273.1 (20), 208.1 (20), 196.1 (40), 117.1 (50), 92.1(100), $77.1(50)$.

(2E)-1-(4-Fluorophenyl)-2-\{1-[4-(methylsulfonyl)phenyl]ethylidene\}hydrazine (3b)

Yield: 68\%; mp: $179{ }^{\circ} \mathrm{C}$; IR (KBr): v $\left(\mathrm{cm}^{-1}\right) 3300(\mathrm{NH}), 1300,1160\left(\mathrm{SO}_{2}\right)$, Mass, $\mathrm{m} / \mathrm{z}$ (\%): 306.2 (M+, 30), 264.5 (20), 227.0 (20), 149.1 (30), 109.1 (100), 83.1 (90).

(2E)-1-(4-chlorophenyl)-2-\{1-[4-(methylsulfonyl)phenyl]ethylidene\}hydrazine (3c)

Yield: $70 \%$; mp: $194-195{ }^{\circ} \mathrm{C}$; IR (KBr): v $\left(\mathrm{cm}^{-1}\right) 3280(\mathrm{NH}), 1310,1150\left(\mathrm{SO}_{2}\right)$, Mass, $m / z(\%): 322.2(\mathrm{M}+, 10), 279.6$ (20), 213.1 (20), 156.1 (30), 138.1 (100), $111.1(50), 77.1(50)$.

(2E)-1-(4-methylphenyl)-2-\{1-[4-(methylsulfonyl)phenyl]ethylidene\}hydrazine (3d)

Yield: $62 \%$; mp: $138-139{ }^{\circ} \mathrm{C}$; IR (KBr): v $\left(\mathrm{cm}^{-1}\right) 3290(\mathrm{NH}), 1300,1150\left(\mathrm{SO}_{2}\right)$, Mass, $m / z(\%): 302.2(\mathrm{M}+, 10), 273.2(30), 222.1(20), 156.1$ (40), $119.2(100), 91.2$ 
(60), $77.1(30)$.

(2E)-1-(4-methoxyphenyl)-2-\{1-[4-(methylsulfonyl)phenyl]ethylidene\}hydrazine (3e)

Yield: 50\%; mp: 142-143 ${ }^{\circ} \mathrm{C}$; IR (KBr): v ( $\left.\mathrm{cm}^{-1}\right) 3300(\mathrm{NH}), 1300,1150\left(\mathrm{SO}_{2}\right)$, Mass, $m / z$ (\%): $318.3(\mathrm{M}+, 10), 303.3$ (20), 239.1 (30), 165.6 (30), 122.2 (80), 92.1 (50), 79.1 (100).

General procedure for preparation of 5-substituted-2-(4-(methyl sulfonyl)phenyl)-1H-indoles (4)

$4 \mathrm{mmol}$ of 3 was added to polyphosphoric acid $(5 \mathrm{~g})$ and the mixture was heated at $120{ }^{\circ} \mathrm{C}$ for $30 \mathrm{~min}$. After this time, the mixture was cooled and poured in $100 \mathrm{ml}$ cold water. The solid product was filtered and boiled with $100 \mathrm{ml}$ of ethanol. The hot mixture is treated with a little decolourising carbon and filtered through a pre-heated Buchner funnel; wash the residue with $40 \mathrm{ml}$ of hot ethanol. Cool the combined filtrates to room temperature, filter off the 2-phenylindole and wash it with $20 \mathrm{ml}$ of cold alcohol. The obtained product was dried and recrystallized from ethanol (Yields: 60-92\%).

\section{2-[4-(Methylsulfonyl)phenyl]-1H-indole (4a)}

Yield: 92\%; mp: 219-220 ${ }^{\circ} \mathrm{C}$; IR (KBr): v ( $\left.\mathrm{cm}^{-1}\right) 3290(\mathrm{NH}), 1300,1150\left(\mathrm{SO}_{2}\right)$; ${ }^{1} \mathrm{H}$ NMR (chloroform-D): $\delta$ (ppm) 3.01 (s, $3 \mathrm{H}, \mathrm{SO}_{2} \mathrm{CH}_{3}$ ), $6.89(\mathrm{~s}, 1 \mathrm{H}$, indole $\mathrm{H}-3$ ), $7.01(\mathrm{~m}, 1 \mathrm{H}$, indole $\mathrm{H}-5), 7.13(\mathrm{~m}, 1 \mathrm{H}$, indole $\mathrm{H}-6), 7.37(\mathrm{~d}, 1 \mathrm{H}$, Indole $\mathrm{H}-4, \mathrm{~J}=8.1$ $\mathrm{Hz}$ ), $7.55(\mathrm{~d}, 1 \mathrm{H}$, indole $\mathrm{H}-7, \mathrm{~J}=8.1 \mathrm{~Hz}), 7.88$ (d, 2H, 4-methylsulfonyl phenyl $\mathrm{H}-2$ \& $\mathrm{H}-6, \mathrm{~J}=8.5 \mathrm{~Hz}$ ), 7.93 (d, 2H, 4-methylsulfonyl phenyl H-3 \& H-5, J = 8.5 Hz), 10.76 (s, 1H, NH) ; Ms: m/z (\%): $271.2\left(\mathrm{M}^{+}, 100\right), 208.1$ (35), 192.2 (95), 165.1 (40), 139.1 (20), 95.7 (30), 89.1 (40). Anal. Calcd. for $\mathrm{C}_{15} \mathrm{H}_{13} \mathrm{NO}_{2} \mathrm{~S}$ : C, 66.40; $\mathrm{H}, 4.83 ; \mathrm{N}, 5.16$. Found: C, 66.65; H, 4.62; N, 5.19.

\section{5-Fluoro-2-[4-(methylsulfonyl)phenyl]-1H-indole (4b)}

Yield: 85\%; mp: $243{ }^{\circ} \mathrm{C}$; IR (KBr): v $\left(\mathrm{cm}^{-1}\right) 3260(\mathrm{NH}), 1300,1160\left(\mathrm{SO}_{2}\right) ;{ }^{1} \mathrm{H}$ 
NMR (chloroform-D): $\delta(\mathrm{ppm}) 3.02\left(\mathrm{~s}, 3 \mathrm{H}, \mathrm{SO}_{2} \mathrm{CH}_{3}\right), 6.84(\mathrm{~s}, 1 \mathrm{H}$, indole $\mathrm{H}-3), 7.09-$ $7.17(\mathrm{~m}, 3 \mathrm{H}$, indole $\mathrm{H}-4, \mathrm{H}-6$ \& $\mathrm{H}-7), 7.89$ (d, 2H, 4-methylsulfonyl phenyl $\mathrm{H}-2$ \& $\mathrm{H}-$ $6, \mathrm{~J}=8.4 \mathrm{~Hz}$ ), 7.90 (d, 2H, 4-methylsulfonyl phenyl H-3 \& H-5, J = 8.4 Hz), 11.06 (s, 1H, NH); Ms: m/z (\%): $289.2\left(\mathrm{M}^{+}, 70\right), 226.2$ (50), 210.2 (100), $198.2(40), 183.2$ (70), 158.2 (30), 107.1 (50), 91.2 (40). Anal. Calcd. for $\mathrm{C}_{15} \mathrm{H}_{12} \mathrm{NO}_{2} \mathrm{FS}: \mathrm{C}, 62.27 ; \mathrm{H}$, 4.18; N, 4.44. Found: C, 62.55; H, 4.32; N, 4.35.

\section{5-Chloro-2-[4-(methylsulfonyl)phenyl]-1H-indole (4c)}

Yield: 88\%; mp: $254{ }^{\circ} \mathrm{C}$; IR ( $\left.\mathrm{KBr}\right): \mathrm{v}\left(\mathrm{cm}^{-1}\right) 3230(\mathrm{NH}), 1300,1150\left(\mathrm{SO}_{2}\right) ;{ }^{1} \mathrm{H}$ NMR (chloroform-D): $\delta$ (ppm) 3.01 (s, 3H, $\left.\mathrm{SO}_{2} \mathrm{CH}_{3}\right), 6.76(\mathrm{~s}, 1 \mathrm{H}$, indole $\mathrm{H}-3), 6.99$ (d, $1 \mathrm{H}$, indole $\mathrm{H}-7, \mathrm{~J}=8.4 \mathrm{~Hz}), 7.25(\mathrm{~d}, 1 \mathrm{H}$, indole $\mathrm{H}-6, \mathrm{~J}=8.4 \mathrm{~Hz}), 7.44(\mathrm{~s}, 1 \mathrm{H}$, indole $\mathrm{H}-4), 7.83$ (d, 2H, 4-methylsulfonyl phenyl $\mathrm{H}-2$ \& $\mathrm{H}-6, \mathrm{~J}=8.5 \mathrm{~Hz}), 7.87$ (d, $2 \mathrm{H}, 4$-methylsulfonyl phenyl $\mathrm{H}-3$ \& $\mathrm{H}-5, \mathrm{~J}=8.5 \mathrm{~Hz}$ ), 11.09 (s, $1 \mathrm{H}, \mathrm{NH}) ; \mathrm{Ms}: \mathrm{m} / \mathrm{z}(\%)$ : $305.1\left(\mathrm{M}^{+}, 40\right), 242.2$ (40), 226.2 (50), 191.2 (100), 163.2 (60), 149.2 (30), 158.2 (30), 95.6 .1 (60), 76.1 (50). Anal. Calcd. for $\mathrm{C}_{15} \mathrm{H}_{12} \mathrm{NO}_{2} \mathrm{ClS}$ : C, 58.92; H, 3.96; N, 4.58. Found: C, 59.15; H, 4.12; N, 4.35.

\section{5-Methyl-2-[4-(methylsulfonyl)phenyl]-1H-indole (4d)}

Yield: $68 \%$; mp: $110-111^{\circ} \mathrm{C}$; IR (KBr): v ( $\left.\mathrm{cm}^{-1}\right) 3270(\mathrm{NH}), 1310,1150\left(\mathrm{SO}_{2}\right)$; ${ }^{1} \mathrm{H}$ NMR (chloroform-D): $\delta(\mathrm{ppm}) 2.28\left(\mathrm{~s}, 3 \mathrm{H}, \mathrm{CH}_{3}\right), 3.03\left(\mathrm{~s}, 3 \mathrm{H}, \mathrm{SO}_{2} \mathrm{CH}_{3}\right), 6.64$ (s, $1 \mathrm{H}$, indole $\mathrm{H}-3$ ), 6.79 (s, $1 \mathrm{H}$, indole $\mathrm{H}-4), 6.86(\mathrm{~d}, 1 \mathrm{H}$, Indole $\mathrm{H}-6, \mathrm{~J}=8.3 \mathrm{~Hz}$ ), 6.99 (d, $1 \mathrm{H}$, indole $\mathrm{H}-7, \mathrm{~J}=8.3 \mathrm{~Hz}$ ), $7.81(\mathrm{~d}, 2 \mathrm{H}, 4$-methylsulfonyl phenyl $\mathrm{H}-2 \mathrm{H} \mathrm{H}-6, \mathrm{~J}=$ $8.5 \mathrm{~Hz}$ ), 7.84 (d, 2H, 4-methylsulfonyl phenyl H-3 \& H-5, J = 8.5 Hz), $10.72(\mathrm{~s}, 1 \mathrm{H}$, $\mathrm{NH}$ ); Ms: $m / z$ (\%): 285.2 (M+, 30), 238.2 (20), 209.1 (20), 183.0 (30), 134.1 (100), 107.1 (40), 92.1 (60), 77.1 (50). Anal. Calcd. for $\mathrm{C}_{16} \mathrm{H}_{15} \mathrm{NO}_{2} \mathrm{~S}: \mathrm{C}, 67.34 ; \mathrm{H}, 5.30 ; \mathrm{N}$, 4.91. Found: C, $67.65 ; \mathrm{H}, 5.12 ; \mathrm{N}, 4.85$.

\section{5-Methoxy-2-[4-(methylsulfonyl)phenyl]-1H-indole (4e)}

Yield: 60\%; mp: $118-120{ }^{\circ} \mathrm{C}$; IR (KBr): v ( $\left.\mathrm{cm}^{-1}\right) 3250(\mathrm{NH}), 1300,1150\left(\mathrm{SO}_{2}\right)$; ${ }^{1} \mathrm{H}$ NMR (chloroform-D): $\delta$ (ppm) $3.03\left(\mathrm{~s}, 3 \mathrm{H}, \mathrm{SO}_{2} \mathrm{CH}_{3}\right.$ ), $3.65\left(\mathrm{~s}, 3 \mathrm{H}, \mathrm{OCH}_{3}\right.$ ), 6.61 (s, $1 \mathrm{H}$, indole $\mathrm{H}-3$ ), $6.74(\mathrm{~s}, 1 \mathrm{H}$, indole $\mathrm{H}-4), 6.81(\mathrm{~d}, 1 \mathrm{H}$, Indole $\mathrm{H}-6, \mathrm{~J}=8.3 \mathrm{~Hz}), 6.97$ 
(d, $1 \mathrm{H}$, indole $\mathrm{H}-7, \mathrm{~J}=8.3 \mathrm{~Hz}$ ), $7.81(\mathrm{~d}, 2 \mathrm{H}, 4$-methylsulfonyl phenyl $\mathrm{H}-2 \mathrm{H} \mathrm{H}-6, \mathrm{~J}=$ $8.5 \mathrm{~Hz}$ ), 7.84 (d, 2H, 4-methylsulfonyl phenyl H-3 \& H-5, J = 8.5 Hz), $10.72(\mathrm{~s}, 1 \mathrm{H}$, $\mathrm{NH})$; Ms: m/z (\%): $301.5\left(\mathrm{M}^{+}, 30\right), 273.7$ (20), 219.5 (30), 183.0 (40), 152.1 (50), 104.0 (100), 89.1 (50). Anal. Calcd. for $\mathrm{C}_{16} \mathrm{H}_{15} \mathrm{NO}_{3} \mathrm{~S}: \mathrm{C}, 63.77 ; \mathrm{H}, 5.02 ; \mathrm{N}, 4.65$. Found: C, 63.95; $\mathrm{H}, 4.82 ; \mathrm{N}, 4.37$.

General procedure for preparation of 5-substituted-2-(4-aminophenyl)-1Hindoles (5)

The compounds $\mathbf{5 a - 5 c}$ were prepared according to the procedure as described for 4 . The obtained products were crystallized from ethanol (Yields: 40 $62 \%)$.

\section{4-(1H-Indol-2-yl)aniline (5a)}

Yield: 60\%; mp: 201-202 ${ }^{\circ} \mathrm{C}$; IR (KBr): v $\left(\mathrm{cm}^{-1}\right)$ 3300, $3250\left(\mathrm{NH}_{2}\right) ;{ }^{1} \mathrm{H}$ NMR (chloroform-D): $\delta$ (ppm) 6.65 (d, 2H, 4-aminophenyl H-3 \& H-5, J = 8.3 Hz), 6.81 (s, $1 \mathrm{H}$, indole $\mathrm{H}-3), 7.01(\mathrm{~m}, 1 \mathrm{H}$, indole $\mathrm{H}-5), 7.12(\mathrm{~m}, 1 \mathrm{H}$, indole $\mathrm{H}-6), 7.30-7.48(\mathrm{~m}$, $2 \mathrm{H}$, indole $\mathrm{H}-4$ \& $\mathrm{H}-7$ ), 7.24 (d, 2H, 4-amino phenyl $\mathrm{H}-2$ \& $\mathrm{H}-6, \mathrm{~J}=8.3 \mathrm{~Hz}$ ), 10.61 (s, $1 \mathrm{H}, \mathrm{NH})$.

\section{4-(5-Fluoro-1H-indol-2-yl)aniline (5b)}

Yield: 43\%; mp: $215-216{ }^{\circ} \mathrm{C}$; IR ( $\left.\mathrm{KBr}\right): v\left(\mathrm{~cm}^{-1}\right) 3310,3260\left(\mathrm{NH}_{2}\right)$;

${ }^{1} \mathrm{H}$ NMR (chloroform-D): $\delta$ (ppm) 6.68 (d, 2H, 4-aminophenyl H-3 \& H-5, J = 8.2 $\mathrm{Hz}), 6.76\left(\mathrm{~s}, 1 \mathrm{H}\right.$, indole $\left.\mathrm{H}_{3}\right), 7.02-7.21(\mathrm{~m}, 3 \mathrm{H}$, indole $\mathrm{H}-4, \mathrm{H}-6$ \& $\mathrm{H}-7), 7.25(\mathrm{~d}, 2 \mathrm{H}$, 4-aminophenyl H-2 \& H-6, J = 8.2 Hz), $10.73(\mathrm{~s}, 1 \mathrm{H}, \mathrm{NH})$.

\section{4-(5-Methoxy-1H-indol-2-yl)aniline (5c)}

Yield: 40\%; mp: $171-172{ }^{\circ} \mathrm{C}$; IR (KBr): v $\left(\mathrm{cm}^{-1}\right)$ 3340, $3250\left(\mathrm{NH}_{2}\right) ;{ }^{1} \mathrm{H}$ NMR (chloroform-D): $\delta$ (ppm) 3.53 (s, 3H, OCH $\left.{ }_{3}\right), 6.55$ (d, 2H, 4-aminophenyl H-3 \& H-5, $\mathrm{J}=8.2 \mathrm{~Hz}), 6.66(\mathrm{~s}, 1 \mathrm{H}$, indole $\mathrm{H}-3), 6.70(\mathrm{~s}, 1 \mathrm{H}$, indole $\mathrm{H}-4), 6.79(\mathrm{~d}, 1 \mathrm{H}$, indole $\mathrm{H}$ $6, \mathrm{~J}=8.3 \mathrm{~Hz}), 6.92(\mathrm{~d}, 1 \mathrm{H}$, indole $\mathrm{H}-7, \mathrm{~J}=8.3 \mathrm{~Hz}), 7.23(\mathrm{~d}, 2 \mathrm{H}, 4$-aminophenyl $\mathrm{H}-2$ \& $\mathrm{H}-6, \mathrm{~J}=8.2 \mathrm{~Hz}), 10.53(\mathrm{~s}, 1 \mathrm{H}, \mathrm{NH})$. 
General procedure for preparation of 5-substituted-2-(4-(azido) phenyl)-1Hindoles (6)

$6 \mathrm{mmol}$ of 5 was added to $10 \mathrm{ml}$ of cold water and $5 \mathrm{ml}$ of hydrochloric acid $(12 \mathrm{~N})$ and the mixture was stirred at r.t. for $1 \mathrm{hr}$. After this time, the mixture was cooled $\left(0{ }^{\circ} \mathrm{C}\right)$ and a solution of $6 \mathrm{mmol}$ sodium nitrite in $5 \mathrm{ml}$ water was added slowly to the mixture. Then $6 \mathrm{mmol}$ sodium azide in $5 \mathrm{ml}$ water was added to reaction medium. The solid product was filtered, washed with water and crystallized from ethanol (Yields: 55-68\%).

\section{2-(4-Azidophenyl)-1H-indole (6a)}

Yield: 68\%; mp: $120-121^{\circ} \mathrm{C}$; IR ( $\left.\mathrm{KBr}\right): \mathrm{v}\left(\mathrm{cm}^{-1}\right) 3270(\mathrm{NH}), 2120\left(\mathrm{~N}_{3}\right) ;{ }^{1} \mathrm{H}$ NMR (chloroform-D): $\delta$ (ppm) 6.85 (s, $1 \mathrm{H}$, indole $\mathrm{H}-3), 7.01$ (m, 1H, indole $\mathrm{H}-5), 7.13(\mathrm{~m}$, $1 \mathrm{H}$, indole $\mathrm{H}-6), 7.32-7.50(\mathrm{~m}, 2 \mathrm{H}$, indole $\mathrm{H}-4$ \& $\mathrm{H}-7), 7.55(\mathrm{~d}, 2 \mathrm{H}, 4$-azidophenyl $\mathrm{H}-2$ \& H-6, J = 8.3 Hz), 7.85 (d, 2H, 4-azidophenyl H-3 \& H-5, J = 8.5 Hz), $10.56(\mathrm{~s}, 1 \mathrm{H}$, $\mathrm{NH})$; Anal. Calcd. for $\mathrm{C}_{14} \mathrm{H}_{10} \mathrm{~N}_{4}$ : C, 71.78; $\mathrm{H}, 4.30 ; \mathrm{N}, 23.92$. Found: C, 71.65; $\mathrm{H}$, 4.02; N, 23.79.

\section{2-(4-Azidophenyl)-5-fluoro-1H-indole (6b)}

Yield: 60\%; mp: $135-136{ }^{\circ} \mathrm{C}$; IR $(\mathrm{KBr}): \mathrm{v}\left(\mathrm{cm}^{-1}\right) 3260(\mathrm{NH}), 2140\left(\mathrm{~N}_{3}\right) ;{ }^{1} \mathrm{H} \mathrm{NMR}$ (chloroform-D): $\delta$ (ppm) 6.80 (s, $1 \mathrm{H}$, indole $\mathrm{H}_{3}$ ), 7.05-7.25 (m, 3H, indole $\mathrm{H}-4, \mathrm{H}-6$ \& $\mathrm{H}-7$ ), 7.56 (d, 2H, 4-azidophenyl H-2 \& H-6, J = 8.4 Hz), 7.88 (d, 2H, 4-azidophenyl $\mathrm{H}-3$ \& $\mathrm{H}-5, \mathrm{~J}=8.4 \mathrm{~Hz}), 10.95(\mathrm{~s}, 1 \mathrm{H}, \mathrm{NH})$; Anal. Calcd. for $\mathrm{C}_{14} \mathrm{H}_{9} \mathrm{~N}_{4} \mathrm{~F}: \mathrm{C}, 66.66 ; \mathrm{H}$, 3.60; N, 22.21. Found: C, 66.85; H, 3.82; N, 22.35 .

\section{2-(4-Azidophenyl)-5-methoxy-1H-indole (6c)}

Yield: 55\%; mp: $70-71{ }^{\circ} \mathrm{C}$; IR ( $\left.\mathrm{KBr}\right): \mathrm{v}\left(\mathrm{cm}^{-1}\right) 3250(\mathrm{NH}), 2110\left(\mathrm{~N}_{3}\right) ;{ }^{1} \mathrm{H}$ NMR (chloroform-D): $\delta$ (ppm) $3.55\left(\mathrm{~s}, 3 \mathrm{H}, \mathrm{OCH}_{3}\right), 6.61(\mathrm{~s}, 1 \mathrm{H}$, indole $\mathrm{H}-3), 6.71(\mathrm{~s}, 1 \mathrm{H}$, indole $\mathrm{H}-4), 6.80(\mathrm{~d}, 1 \mathrm{H}$, indole $\mathrm{H}-6, \mathrm{~J}=8.2 \mathrm{~Hz}), 6.95(\mathrm{~d}, 1 \mathrm{H}$, indole $\mathrm{H}-7, \mathrm{~J}=8.2 \mathrm{~Hz}$ ), 7.60 (d, 2H, 4-azidophenyl H-2 \& H-6, J = $8.4 \mathrm{~Hz}$ ), 7.80 (d, 2H, 4-azidophenyl H-3 \& $\mathrm{H}-5, \mathrm{~J}=8.4 \mathrm{~Hz}$ ), $10.72(\mathrm{~s}, 1 \mathrm{H}, \mathrm{NH})$; Anal. Calcd. for $\mathrm{C}_{15} \mathrm{H}_{12} \mathrm{~N}_{4} \mathrm{O}: \mathrm{C}, 68.17 ; \mathrm{H}, 4.58$; N, 21.20. Found: C, 68.45; H, 4.72; N, 21.36. 


\section{In vitro cyclooxygenase (COX) inhibition assays}

The ability of the test compounds listed in Table 1 to inhibit ovine COX-1 and COX-2 ( $\mathrm{IC}_{50}$ value, $\left.\mu \mathrm{M}\right)$ was determined using chemiluminescent enzyme assays kit (catalog number 560101, Cayman Chemical, Ann Arbor, MI, USA) according to our previously reported method [13].

\section{Molecular modeling (docking) studies}

Docking studies were performed using Autodock software Version 3.0. The coordinates of the X-ray crystal structure of the selective COX-2 inhibitor SC-558 bound to the murine COX-2 enzyme was obtained from the RCSB Protein Data Bank (1cx2) and hydrogens were added. The ligand molecules were constructed using the Builder module and were energy minimized for 1000 iterations reaching a convergence of $0.01 \mathrm{kcal} / \mathrm{mol} \AA$. The energy minimized ligands were superimposed on SC-558 in the PDB file 1cx2 after which SC-558 was deleted. The purpose of docking is to search for favorable binding configuration between the small flexible ligands and the rigid protein. Protein residues with atoms greater than $7.5 \AA$ from the docking box were removed for efficiency. Searching is conducted within a specified 3D docking box using annealing based on the Monte Carlo method and MMFF94 molecular mechanics force field for 8000 iterations. These docked structures were very similar to the minimized structures obtained initially. The quality of the docked structures was evaluated by measuring the intermolecular energy of the ligand-enzyme assembly [19].

\section{References}

[1] Fu JY, Masferrer JL, Seibert K, Raz A, Needleman P. The induction and suppression of prostaglandin $\mathrm{H} 2$ synthase (cyclooxygenase) in human monocytes. J Biol Chem. 1990; 265: 16737-16736. 
[2] Smith WL, DeWitt DL.

Prostaglandin endoperoxide $\mathrm{H}$ synthases-1 and -2 .

Adv Immunol.1996; 62: 167-215.

[doi:10.1016/S0065-2776(08)60430-7]

[3] Herschman HR.

Prostaglandin synthase 2 .

Biochem Biophys Acta.1996; 1299: 125-140.

[4] Kawamori T, Rao CV, Seibert K, Reddy BS.

Chemopreventive activity of celecoxib, a specific cyclooxygenase-2 inhibitor, against colon carcinogenesis.

Cancer Res. 1998; 58: 406-412.

[5] Katori M, Majima M.

Cyclooxygenase-2: its rich diversity of roles and possible application of its selective inhibitors.

Inflamm Res. 2000; 49: 367-392.

[doi:10.1007/s000110050605]

[6] Vane JR, Botting RM.

Anti-inflammatory drugs and their mechanism of action.

Inflamm Res. 1998; 47: S78-S87.

[doi:10.1007/s000110050284]

[7] Penning TD, Tally JJ. Bertenshaw SR, Carter JS, Collins PW, Docter S, Graneto MJ, Lee LF, Malecha JW, Miyashiro JM, Rogers R, Rogier DJ, Yu SS, Anderson GD, Burton EG, Cogburn JN, Gregory SA, Koboldt CM, Perkins WE, Seibert K, Veenhuizen AW, Zhang YY, Isakson PC.

Synthesis and biological evaluation of the 1,5-diarylpyrazole class of cyclooxygenase-2 inhibitors: identification of 4-[5-(4-methylphenyl)-3(trifluoromethyl)-1H-pyrazol-1-yl]benzenesulfonamide (SC-58635, celecoxib). J Med Chem. 1997; 40: 1347-1365.

[doi:10.1021/jm960803q]

[8] Riendeau D, Percival MD, Brideau C, Dube CS, Ethier D, Falgueyret JP, Friesen RW, Gordon R, Greig G, Guay J, Girard Y, Prasit P, Zamboni R, Rodger IW, Gresser M, Ford-Hutchinson A, Young RN, Chan CC.

Etoricoxib (MK-0663): preclinical profile and comparison with other agents that selectively inhibit cyclooxygenase-2.

J Pharmacol ExpTher. 2002; 296: 558-566. 
[9] Prasit P, Wang Z, Brideau C, Chan CC, Charlson S, Cromlish W, Ethier D, Evans JF, Ford-Hutchinson AW, Gauthier JY, Gordon R, Guay J, Gresser M, Kargman S, Kennedy B, Leblanc Y, Leger S, Mancini JO, Neil GP, Quellet M, Percival MD, Perrier H, Riendeau D, Rodger I, Tagari P, Therien M, Vikers P, Wong E, Xu L, Young RN, Zamboni R, Boyce S, Rupniak N, Forrest M, Visco $D$, Patrick D.

The discovery of rofecoxib, [MK 966, Vioxx, 4-(4'-methylsulfonylphenyl)-3phenyl-2(5H)-furanone], an orally active cyclooxygenase-2-inhibitor.

Bioorg Med Chem Lett. 1999; 9: 1773-1778.

[doi:10.1016/S0960-894X(99)00288-7]

[10] Friesen RW, Dube D, Fortin R, Frenette R, Prescott S, Cromlish W, Greig GM, Kargman S, Wong E, Chan CC, Gordon R, Xu L, Young RN.

2-Pyridinyl-3-(4-methylsulfonyl)phenylpyridines: selective and orally active cyclooxygenase-2 inhibitors.

Bioorg Med Chem Lett. 1998; 6: 2777-2782.

[doi:10.1016/S0960-894X(98)00499-5]

[11] Talley JJ, Brown DL, Carter JS, Graneto MJ, Koboldt CM, Masferrer JL, Perkins WE, Rogers RS, Shaffer AF, Zhang YY, Zweifel BS, Seibert K.

$\mathrm{N}$-[[(5-methyl-3-phenylisoxazol-4-yl)-phenyl]sulfonyl]propanamide, sodium salt, parecoxib sodium: A potent and selective inhibitor of COX-2 for parenteral administration.

J Med Chem. 2000; 43: 775-777.

[doi:10.1021/jm990577v]

[12] Zarghi A, Rao PNP, Knaus EE.

Design and synthesis of new rofecoxib analogs as selective cyclooxygenase-2 (COX-2) inhibitors: replacement of the methanesulfonyl pharmacophore by a $\mathrm{N}$-acetylsulfonamido bioisostere.

J Pharm Pharm Sci. 2007; 10: 159-167.

[13] Zarghi A, Najafnia L, Daraie B, Dadrass OG, Hedayati M.

Synthesis of 2,3-diaryl-1,3-thiazolidine-4-one derivatives as selective cyclooxygenase (COX-2) inhibitors.

Bioorg Med Chem Lett. 2007; 17: 5634-5637.

[doi:10.1016/j.bmcl.2007.07.084]

[14] Zarghi A, Arfaee S, Rao PNP, Knaus EE.

Design, synthesis, and biological evaluation of 1,3-diarylprop-2-en-1-ones : a novel class of cyclooxygenase-2 inhibitors.

Bioorg Med Chem. 2006; 14: 2600-2605.

[doi:10.1016/j.bmc.2005.11.041] 
[15] Zarghi A, Zebardast T, Hakimion F, Shirazi FH, Rao PNP, Knaus EE. Synthesis and biological evaluation of 1,3-diphenylprop-2-en-1-ones possessing a methanesulfonamido or an azido pharmacophore as cyclooxygenase-1/-2 inhibitors.

Bioorg Med Chem. 2006; 14: 7044-7050.

[doi:10.1016/j.bmc.2006.06.022]

[16] Zarghi A, Rao PNP, Knaus EE.

Sulfonamido, azidosulfonyl and $\mathrm{N}$-acetylsulfonamido analogues of rofecoxib:4[4-(N-acetylsulfonamido)phenyl]-3-(4-methanesulfonyl phenyl)-2(5H)furanone is a potent and selective cyclooxygenase-2 inhibitor.

Bioorg Med Chem Lett. 2004; 14: 1957-1960.

[doi:10.1016/j.bmcl.2004.01.076]

[17] Homes TP, Mattner F, Keller PA, Katsifis A.

Synthesis and in vitro binding of N,N-dialkyl-2-phenylindol-3-yl-glyoxylamides for the peripheral benzodiazepine binding sites.

Bioorg Med Chem. 2006; 14: 3938-3946.

[doi:10.1016/j.bmc.2006.01.039]

[18] Gungor T, Teulon JM.

Preparation of 1,2-diarylindoles as COX-2 inhibitors.

WO 9805639. 1998.

[19] Kurumbail RG, Stevens AM, Gierse JK, McDonald JJ, Stegeman RA, Pak JY, Gildehaus D, Miyashiro JM, Penning TD, Seibert K, Isakson PC, Stallings WC. Structural basis for selective inhibition of cyclooxygenase-2 by antiinflammatory agents.

Nature. 1996; 384: 644-648.

[doi:10.1038/384644a0]

Received May $27^{\text {th }}, 2008$

Accepted (after revision) July $7^{\text {th }}, 2008$

Available online at www.scipharm.at July $8^{\text {th }}, 2008$ 